\title{
Topological instabilities in ac-driven bosonic systems
}

\author{
G. Engelhardt ${ }^{1}$ * M. Benito ${ }^{2}$, G. Platero ${ }^{2}$, and T. Brandes ${ }^{1}$ \\ ${ }^{1}$ Institut für Theoretische Physik, Technische Universität Berlin, Hardenbergstr. 36, 10623 Berlin, Germany \\ ${ }^{2}$ Instituto de Ciencia de Materials de Madrid, CSIC, 28049 Madrid, Spain
}

\begin{abstract}
Under non-equilibrium conditions, bosonic modes can become dynamically unstable with an exponentially growing occupation. On the other hand, topological band structures give rise to symmetry protected midgap states. In this letter, we investigate the interplay of instability and topology. Thereby, we establish a general relation between topology and instability under ac-driving. We apply our findings to create dynamical instabilities which are strongly localized at the boundaries of a finite-size system. As these localized instabilities are protected by symmetry, they can be considered as topological instabilities.
\end{abstract}

PACS numbers: $05.30 . J p, 63.20 . P \mathrm{w}, 67.85 .-\mathrm{d}, 42.50 . \mathrm{Dv}$

Introduction. Due to their underlying symplectic structure, bosonic systems can exhibit so-called dynamical instabilities [1]. This effect can occur in the presence of non-particle conserving terms which appear, e.g., in the Bogoliubov excitations of Bose-Einstein condensates. Thereby, the bosonic occupation of a mode grows exponentially in time due to a non-equilibrium state of the system. On the other hand, the theory of topological band structures predicts symmetry protected midgap states [4 13. A priory, dynamical instability and topology are independent phenomena.

In this letter, we formalize a relation between instabilities and topology under ac driving. More precisely, we demonstrate that different topological phases are always separated by regions of instability. Using this relation, we demonstrate how to employ topology to systematically engineer topologically protected dynamical instabilities. Thereby, spatially localized midgap modes are rendered dynamically unstable with exponentially growing bosonic occupation as has been exemplary proposed for Bose-Einstein condensates in Refs. [11, 14, 15], and for photonic systems [16]. Here, we suggest a very flexible tool in the form of ac-fields in order to engineer topological instabilities governed by corresponding artificial, effective Hamiltonians [17. This simultaneously provides the possibility to detect the midgap states as their occupation increases exponentially in time. Topological instabilities are an effect with no direct analogue in fermionic topological insulators. This stresses the need for a more intensive investigation of topological effects in bosonic systems.

In fermionic systems, ac-driving has been applied to control topological phases 18 24. In particular, the topology of a band can change if there is a degeneracy of the form $\epsilon_{i^{\prime}}=\epsilon_{i}+\Omega$, where $\Omega$ denotes the driving frequency. As this is a single-particle effect, it can also appear in bosonic systems [25, 26]. The main challenge, however, is that dynamical instabilities appearing in bosonic systems generated by an ac-driving con-

\footnotetext{
* georg@itp.tu-berlin.de
}

stitute an obstruction in the search for a stable system with non-trivial topology [25]: by slightly changing parameters, the system might get unintentionally unstable within the bulk, which obscures the existence of midgap states. For the one-dimensional Hamiltonian under consideration, we show how to employ stability diagrams to facilitate the search of adequate system parameters. The latter can be regarded as a higher-dimensional version of the famous Arnold tongues in parametrically driven oscillators [3].

Selective enhancement of edge states and related effects can be achieved using different approaches, e.g., non-Hermitian Hamiltonians [27 30]. Furthermore, in a driven spin chain the crossing of a topological phase transitions is accompanied by a Kibble-Zurek scaling phenomenon [31. These effects raise the question, to what extend the instability-topology relation established here for bosonic ac-driven systems can be generalized to other fields of physics.

The system. Bogoliubov Hamiltonians are important in many areas of physics. For instance, they appear by an expansion of Hamiltonians describing interacting bosonic particle or polariton condensates in orders of the fluctuations [1, 2, 14, 32. They also describe excitations in magnonic crystals [8, 9] or in quantum-optical systems [33. More generally, they appear in the linear stability analysis of nonlinear bosonic systems.

We analyze a one-dimensional system of coupled bosonic modes which is subjected to periodic driving. However, we emphasize that the relation between instability and topology established here apply also for higher-dimensional systems. A Hamiltonian allowing for a systematic investigation of the topological instabilities which we are interested in reads

$$
\begin{aligned}
H & =\sum_{m}-\left(\nu(t) \hat{a}_{m, 1}^{\dagger} \hat{a}_{m, 2}+\nu^{\prime}(t) \hat{a}_{m, 2}^{\dagger} \hat{a}_{m+1,1}+\text { h.c. }\right) \\
& +g \sum_{m, s=\{1,2\}}\left(\hat{a}_{m, s}^{\dagger} \hat{a}_{m, s}^{\dagger}+\text { h.c. }\right)-\sum_{m, s=\{1,2\}} \mu \hat{a}_{m, s}^{\dagger} \hat{a}_{m, s},
\end{aligned}
$$

where $\hat{a}_{m, s}$ with $s=1,2$ are bosonic annihilation operators, $\nu(t) \equiv \nu_{0}+\nu_{1} \cos (\Omega t), \nu^{\prime}(t) \equiv \nu_{0}^{\prime}+\nu_{1}^{\prime} \cos (\Omega t)$ 
a)
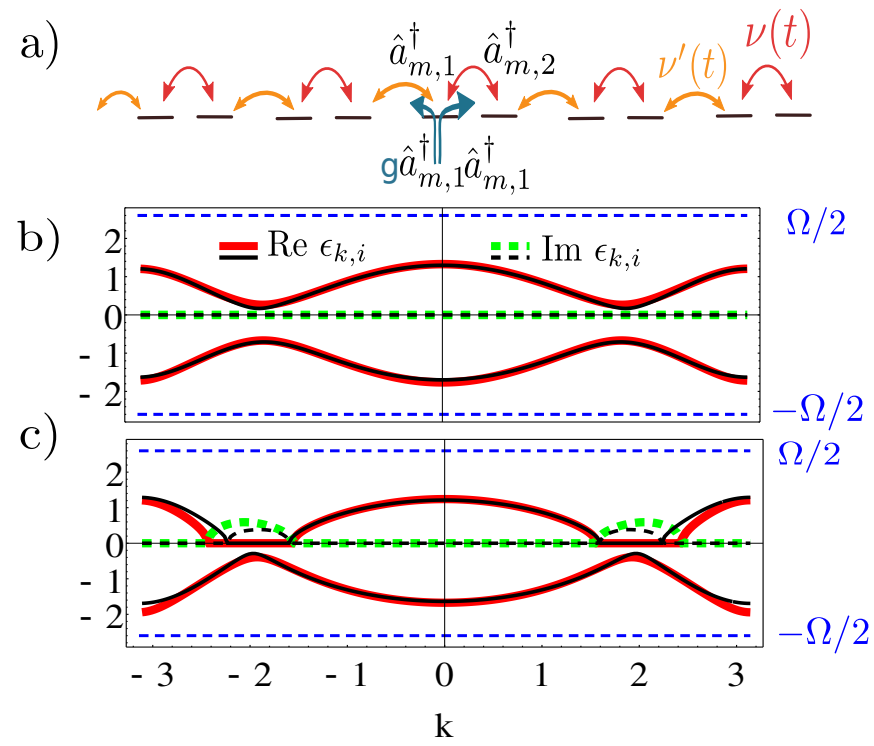

FIG. 1. (Color online) (a) Sketch of the system. (b) and (c) depict quasienergy spectra with colored lines for $\nu_{0}=1.5$, $\nu_{0}^{\prime}=0, \nu_{1}=3, \mu=-5$ and $\Omega=5.2$. We choose $\nu_{1}^{\prime}=11$ and $\nu_{1}^{\prime}=6$ in (b) and (c), respectively. Black lines depict the spectrum of an effective Hamiltonian [35. All quantities are expressed in units of $g$.

and $\mu$ the chemical potential. The system is sketched in Fig. 1(a). For $\nu_{1}=\nu_{1}^{\prime}=0$ the first line resembles the famous Su-Schrieffer-Heeger model which exhibits a topological phase transition for $\nu_{0}=\nu_{0}^{\prime}$ [34. Due to the non-particle-conserving terms in the second line the bosonic modes can exhibit dynamical instabilities with exponentially growing bosonic occupation.

After a transformation into the momentum space, the Bogoliubov Hamiltonian reads

$$
\hat{H}^{(B)}(t)=\frac{1}{2} \sum_{k}\left(\hat{\mathbf{a}}_{k}^{\dagger}, \hat{\mathbf{a}}_{-k}\right) \mathbf{H}_{k}(t)\left(\begin{array}{c}
\hat{\mathbf{a}}_{k} \\
\hat{\mathbf{a}}_{-k}^{\dagger}
\end{array}\right) .
$$

The symbol $\hat{\mathbf{a}}_{k}^{\dagger}=\left(\hat{a}_{k, 1}^{\dagger}, \hat{a}_{k, 2}^{\dagger}\right)$ denotes a vector of bosonic creation operators, and

$$
\mathbf{H}_{k}(t) \equiv \mathbb{1} \otimes \vec{h}(k, t) \vec{\sigma}-\mu \mathbb{1} \otimes \mathbb{1}+g \sigma_{x} \otimes \mathbb{1},
$$

where $\vec{\sigma}=\left(\sigma_{x}, \sigma_{y}\right)$ is a vector of Pauli matrices and we defined a pseudo magnetic-field vector with components $h_{x}(k, t)=-\nu(t)-\nu^{\prime}(t) \cos k \equiv h_{x, 0}(k)+h_{x, 1}(k) \cos \Omega t$ and $h_{y}(k, t)=-\nu^{\prime}(t) \sin k \equiv h_{y, 0}(k)+h_{y, 1}(k) \cos \Omega t$.

Importantly, the Bogoliubov Hamiltonian fulfills a generalized chiral symmetry at all times $t$. This is defined by

$$
\begin{aligned}
& \boldsymbol{\Sigma}\left[\mathbf{H}_{k}(t)+\mu \mathbb{1} \otimes \mathbb{1}-g \sigma_{x} \otimes \mathbb{1}\right] \boldsymbol{\Sigma} \\
& \quad=-\left[\mathbf{H}_{k}(t)+\mu \mathbb{1} \otimes \mathbb{1}-g \sigma_{x} \otimes \mathbb{1}\right],
\end{aligned}
$$

where $\boldsymbol{\Sigma}=\sigma_{z} \otimes \sigma_{z}$. Accordingly, the Hamiltonian corresponds to the topological class BDI according to the
Altland-Zirnbauer classification [36]. The band structure of the undriven and non-interacting system exhibits two bands. They are described by a topological quantum number given by

$$
W=\frac{1}{2 \pi i} \int_{-\pi}^{\pi} \frac{d}{d k} \ln \left[h_{x}(k)+i h_{y}(k)\right],
$$

which counts how often the vector $\vec{h}(k)$ winds around $\vec{h}(k)=0$. Consequently, $W$ can only change, if there is a degeneracy as this is related to $\vec{h}(k)=0$. By definition, the winding number describes translational invariant systems. However, there is an important consequence for finite-sized systems with boundaries. There are spatially confined states close to the boundary with energy located within the band gap. The number of these states equals $W$ [6, 37]. We now show how the physics is modified in the presence of periodic driving and interactions.

Floquet-Bogoliubov Theory. As regular Bogoliubov excitation energies of an undriven system, the FloquetBogoliubov quasienergies for bosons reveal the stability of a system. It is stable, if all quasienergies are realvalued and unstable if one or more have a finite imaginary part. They can be obtained in analogy to the undriven case [1, 2, 25, 38, 39]: first, we have to solve the differential equation

$$
i \frac{d}{d t} \mathbf{U}(t)=\boldsymbol{\sigma}_{\boldsymbol{z}} \mathbf{H}(t) \mathbf{U}(t), \quad \mathbf{U}(0)=\mathbf{1},
$$

where $\boldsymbol{\sigma}_{\boldsymbol{z}}=\sigma_{z} \otimes \mathbb{1}$ emerges as the Bogoliubov Hamiltonian couples bosonic creation and annihilation operators, whose equation of motion differ by a minus sign. The matrix $\mathbf{U}(2 \pi / \Omega)$ is the Floquet operator and its eigenvalues and eigenstates fulfill

$$
\mathbf{U}(2 \pi / \Omega)\left|\Psi_{i}\right\rangle=e^{-i \frac{2 \pi}{\Omega} \epsilon_{i}}\left|\Psi_{i}\right\rangle,
$$

where $\epsilon_{i}$ denotes the Bogoliubov quasienergies and $\left|\Psi_{i}\right\rangle$ the stroboscopic Floquet states [40. There are always $2 d$ Floquet states where $d$ denotes the dimension of the single-particle Hamiltonian. As usual quasienergies, the real part of the Bogoliubov quasienergies can be represented within the window $\left(-\frac{\Omega}{2}, \frac{\Omega}{2}\right)$. A system is only stable if the quasienergies for all $k \in(-\pi, \pi)$ are realvalued. In this case we denote the system to be globally stable.

Additionally, we introduce the concept of strong stability according to Refs. [3, 41. A Floquet state with $\operatorname{Im} \epsilon_{i}=0$ is denoted to be strongly stable, if a small perturbation of the system does not result in a finite imaginary part $\operatorname{Im} \epsilon_{i} \neq 0$. If a state is strongly stable, then it can be normalized as $C_{i} \equiv\left\langle\Psi_{i}\left|\boldsymbol{\sigma}_{\boldsymbol{z}}\right| \Psi_{i}\right\rangle= \pm 1$ [1, 41]. For every state with $\epsilon_{i}$ there is a corresponding state with $\epsilon_{i^{\prime}}=-\epsilon_{i}$. If the states are normalizable, then $C_{i}=-C_{i^{\prime}}$. A system is considered to be strongly stable, if all Floquet states are strongly stable.

Two Bogoliubov quasienergy dispersions are depicted in Fig. 1.(b) and (c), where we take the state with $C_{k, i}=1$ 
a)

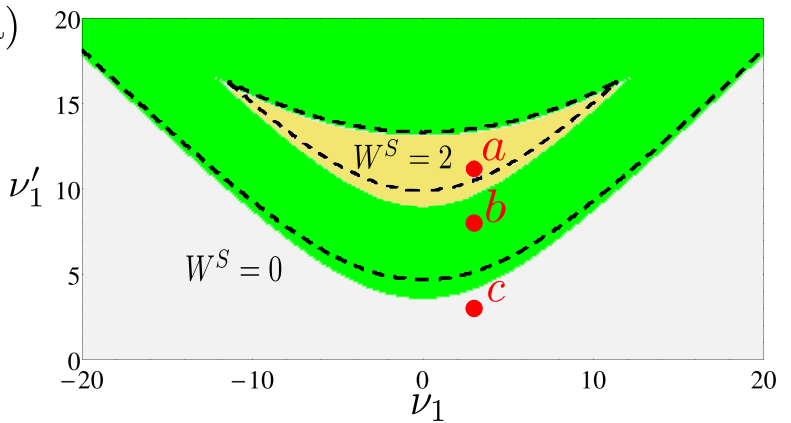

b)

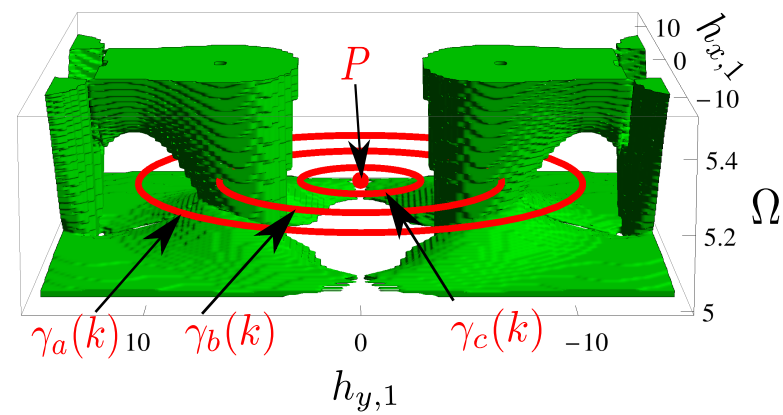

FIG. 2. (Color online) (a) Topological phase diagram. The parameters are as in Fig. 1 (b). In the green areas, the system is not globally stable, so the topological invariant $W^{S}$ in Eq. (9) is not defined there. In the gray and yellow areas, we find that $W^{S}=0$ and $W^{S}=2$, respectively. The topological phases are separated by instability areas (green). Dashed lines are obtained by using an effective Hamiltonian [35. (b) Stability diagram as a function of $h_{x, 1}$ and $h_{y, 1}$ corresponding to (a). The parameters of the curves $\gamma_{a, b, c}$ are depicted in (a) by the points $p=a, b, c$. The parameters $p=a$ and $p=b$ correspond to Fig. 11.b) and (c). Curve $\gamma_{c}$ can be contracted to point $P$, so that it is topologically trivial according to the explanations in the main text.

if it is normalizable. In panel (c), we recognize momenta $k$ with $\operatorname{Im} \epsilon_{k, i} \neq 0$, leading to a dynamical instability with bosonic occupations growing exponentially in time [1, 2], which we analyze in Fig. 2(a), where the system is not globally stable in the green areas.

Following Ref. 3, 41, one finds that if the quasienergies of two strongly stable states with $C_{i} \neq C_{j}$ merge by varying system parameters, thus they become

$$
\operatorname{Re} \epsilon_{i}=\operatorname{Re} \epsilon_{j} \bmod \Omega,
$$

then the states are not strongly stable. Moreover, even when getting unstable, the states $i, j$ still fulfill Eq. (8) which thus constitutes a necessary instability condition. Consequently, if the quasienergy of an originally strongly stable state gets $\operatorname{Re} \epsilon_{i}=0, \Omega / 2$, it is not strongly stable. We relate this to topology in the following.

Topology. Motivated by 8,10 , we define a topological invariant generalizing Eq. (5) for driven bosonic Bogoliubov systems.

$$
W^{S} \equiv \frac{1}{\pi i} \sum_{i \in \mathcal{S}} \int_{-\pi}^{\pi} d k\left\langle\Psi_{k, i}\left|\boldsymbol{\sigma}_{z} \frac{d}{d k}\right| \Psi_{k, i}\right\rangle,
$$

where $\mathcal{S}=\left\{i \mid 0<\epsilon_{k, i}<\Omega / 2 \wedge C_{k, i}=1\right.$ for all $\left.k\right\}$ is the set of all positive normalizable quasienergies. We note that $W^{S}$ is only defined for globally strongly stable systems.

As our Hamiltonian fulfills a generalized chiral symmetry, the topological invariant is integer valued, i.e., $W^{S} \in \mathbb{Z}$. It predicts midgap states energetically located close to $\epsilon_{i}=0, \Omega / 2$, thus, close to the instability condition (8). The number of midgap states at each boundary equals $W^{S}$.

The topological phase diagram is depicted in Fig. 2(a). There we find a phase with $W^{S}=0$ (gray) and one with $W^{S}=2$ (yellow). Interestingly, these two phases are separated by instability regions which is a general feature in driven bosonic systems, cf. below.

Instability-topology relation. We are now in a position to establish a general relation between topology and instability of a bosonic system under ac-driving: The topological invariant, Eq. (9), can only change by a smooth variation of system parameters $p(t)$ with $t \in[0,1]$, if the system is not globally strongly stable for at least one $t=t_{0}$.

The relation is a direct consequence of the instability condition Eq. (8). When we only perform parameter variations so that the system is globally strongly stable, Eq.(8) is never fulfilled and $\mathcal{S}$ remains unchanged. Moreover, the bands $i \in \mathcal{S}$ are not in contact with the bands $i \neq \mathcal{S}$ so that topological invariant can not change.

By definition, in the vicinity of stable but not strongly stable parameters there are always unstable parameters. Consequently, the topological phases in Fig. 2(a) are separated by unstable regions.

We emphasize that this relation is valid for systems of arbitrary dimensions. One only has to replace $W^{S}$ in Eq. (9) by a higher-dimensional topological invariant.

To elucidate this relation, we investigate the stability of the Hamiltonian (2) as a function of $h_{\eta, 1}$ with $\eta=x, y$ and $\Omega$. The result is depicted in Fig. 2(b), where we depict unstable parameters in green. A set of system parameters $p$ specifies a curve as a function of monentum $\mathrm{k}$ in the stability diagram due to the parametrization of $h_{\eta, 1}$ with $\eta=x, y$ below Eq.(3). For instance, the points $p=a, b, c$ depicted in Fig. 2(a) correspond to curves $\gamma_{p}(k)$ in the stability diagram in (b). The curves $\gamma_{a}$ and $\gamma_{c}$ do not traverse any unstable areas, so they are globally stable, while $\gamma_{b}$ is not.

If one can smoothly contract a globally strongly stable $\gamma(k)$ while clearly avoiding areas of unstable parameters, then $W_{\gamma}^{S}=0$. In Fig. 2(b), $\gamma_{c}(k)$ can be trivially contracted to $P$ so that it has a trivial topology. By contrast, the deformation of $\gamma_{a}(k)$ onto a stable point in Fig. 2(b), is only possible by traversing the two unstable green regions. For this reason, it is a canditate for a nontrivial topology with $W_{\gamma}^{S} \neq 0$. We thus have a one-to-one correspondence between points in the topological phase diagram Fig. 2(a), and (non) contractible curves in the stability diagram in Fig. 2(b).

Moreover, the stability diagram assists to find pa- 

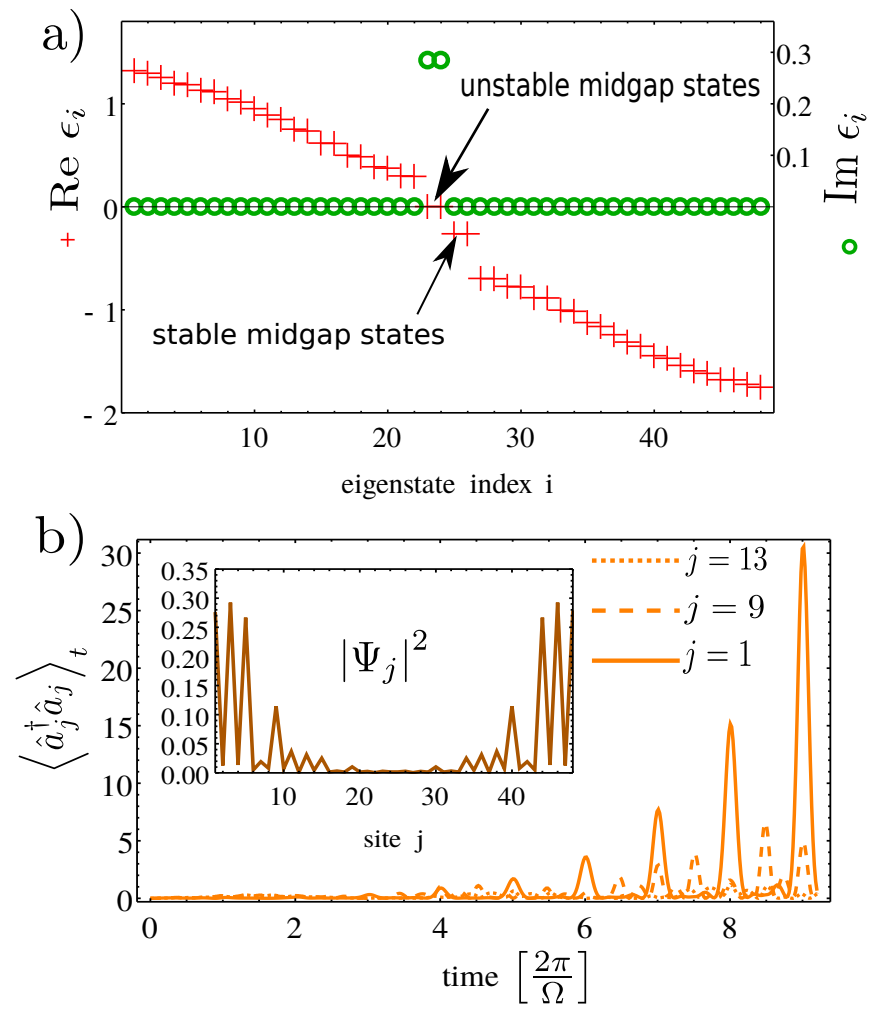

FIG. 3. (Color online) (a) Quasienergy spectrum for a finitesized system with boundaries corresponding to $\gamma_{a}$. There are four midgap states located near $\operatorname{Re} \epsilon_{i}=0$ which we mark with arrows. The imaginary part of two of the midgap states is finite, which renders these states unstable. States not marked by the arrows extend within the bulk and are stable. (b) Time evolution of the occupation of the sites $j=1,9,13$. The initial state is the vacuum state. In the inset we depict one of the unstable midgap states which are responsible for the exponential growth as seen in (b). It is strongly confined close to the boundaries. Note that the site number is $j=2 m+s$, with $m, s$ defined in Eq. (1).

rameters corresponding to a stable and topologicalnontrivial system. We only have to calculate $W^{S}$ for non-contractible curves which can not be transformed to each other in a stable way.

The black lines in Figs. 11(b), (c) and 2(a) depict the calculations using a time-independent effective Hamiltonian [35. For its derivation, we generalized the procedure of Ref. 23. to Bogoliubov Hamiltonians which, to our knowledge, has not been done before. As the effective Hamiltonian resembles the features of the spectrum, it is an appropriate tool to calculate stability diagrams which enable an efficient search for parameters of stable and topologically non-trivial systems.

Topological instabilities. Due to its definition, $W^{S}$ predicts midgap states energetically located close to $\epsilon_{i}=$ $0, \Omega / 2$, thus, close to the instability condition (8). In Fig. 3(a), we depict the numerical quasienergy spectrum for a finite-sized system with boundaries with parameters given by $p=a$ in Fig. 2(a). There are four midgap states with $\operatorname{Re} \epsilon_{i} \approx 0$ which we mark with arrows. Their wave functions are strongly confined to the boundaries. The wave function of one of them is depicted in the inset of panel (b). The imaginary part of the quasienergies of two midgap states is finite so they are dynamically unstable, which gives rise to an exponential growth of the occupation as a function of time. The imaginary part of the quasienergies of the other two midgap states is zero so they are stable. The states not marked by an arrow are bulk modes and are stable.

In principle, one can render all four midgap states to be unstable by slightly adjusting the system parameters as $\operatorname{Re} \epsilon_{i} \approx 0$. However, we did not find parameters where all four midgap states are unstable without destabilizing the bulk modes.

The initial state of the time evolution in Fig. 3(b) is the vacuum state defined by $\hat{a}_{j}|\mathrm{vac}\rangle=0$ [11, 14, 15]. We recognize, that the occupation grows exponentially on sites close to the boundary, while it remains small within the bulk. As the instabilities are generated by the unstable midgap states, they can be thus considered to be topologically protected instabilities.

To conclude, we found that topological phases are separated by regions of instability in ac-driven systems which we illustrated using stability diagrams. We recall that this finding is valid for systems of arbitrary dimension. We used this to selectively generate dynamical instabilities which are strongly localized close to the boundaries. To this end, we employed localized midgap states whose occupation grows exponentially in time. Recently, stability and the onset of chaos have been experimentally explored in a periodically-driven two-mode Bose-Einstein condensate [42. We assume that the spatially extended system investigated here is also a candidate for such an experimental investigation.

Acknowledgments The authors gratefully acknowledge financial support from the DFG Grants No. BR 1528/7, No. BR 1528/8, No. BR 1528/9, No. SFB 910 and No. GRK 1558. This work was supported by the Spanish Ministry through Grant No. MAT2014-58241-P.
[1] Y. Kawaguchi and M. Ueda, Phys. Rep. 520, 253 (2012)

[2] L. Goren, E. Mariani, and A. Stern, Phys. Rev. A 75, 063612 (2007)

[3] V. I. Arnold, Mathematical methods of classical mechanics, Vol. 60 (Springer Science \& Business Media, 1989).

[4] B. A. Bernevig and T. L. Hughes, Topological insula- tors and topological superconductors (Princton University Press, 2013).

[5] L. Fu and C. L. Kane, Phys. Rev. B 74, 195312 (2006)

[6] M. Z. Hasan and C. L. Kane, Rev. Mod. Phys. 82, 3045 (2010).

[7] G. Jotzu, M. Messer, R. Desbuquois, M. Lebrat, 
T. Uehlinger, D. Greif, and T. Esslinger, Nature (London) 515, 237 (2014).

[8] R. Shindou, R. Matsumoto, S. Murakami, and J.-I. Ohe, Phys. Rev. B 87, 174427 (2013)

[9] R. Shindou, J.-I. Ohe, R. Matsumoto, S. Murakami, and E. Saitoh, Phys. Rev. B 87, 174402 (2013).

[10] G. Engelhardt and T. Brandes, Phys. Rev. A 91, 053621 (2015)

[11] S. Furukawa and M. Ueda, New J. Phys 17, 115014 (2015).

[12] C.-E. Bardyn, T. Karzig, G. Refael, and T. C. H. Liew, Phys. Rev. B 93, 020502 (2016)

[13] V. Peano, M. Houde, C. Brendel, F. Marquardt, and A. A. Clerk, arXiv: 1508.01383.

[14] R. Barnett, Phys. Rev. A 88, 063631 (2013)

[15] B. Galilo, D. K. K. Lee, and R. Barnett, Phys. Rev. Lett. 115, $245302(2015)$

[16] V. Peano, M. Houde, F. Marquardt, and A. A. Clerk, arXiv:1604.04179.

[17] V. M. Bastidas, C. Emary, B. Regler, and T. Brandes, Phys. Rev. Lett. 108, 043003 (2012).

[18] M. C. Rechtsman, J. M. Zeuner, Y. Plotnik, Y. Lumer, D. Podolsky, F. Dreisow, S. Nolte, M. Segev, and A. Szameit, Nature (London) 496, 196 (2013).

[19] Y. H. Wang, H. Steinberg, P. Jarillo-Herrero, and N. Gedik, Science 342, 453 (2013).

[20] T. Oka and H. Aoki, Phys. Rev. B 79, 081406 (2009).

[21] A. Kundu and B. Seradjeh, Phys. Rev. Lett. 111, 136402 (2013)

[22] P. Wang, Q.-f. Sun, and X. C. Xie, Phys. Rev. B 90, 155407 (2014)

[23] M. Benito, A. Gómez-León, V. M. Bastidas, T. Brandes, and G. Platero, Phys. Rev. B 90, 205127 (2014).

[24] T. Mikami, S. Kitamura, K. Yasuda, N. Tsuji, T. Oka, and H. Aoki, arXiv: 1511.00755 (2015).

[25] G. Salerno, T. Ozawa, H. M. Price, and I. Carusotto,
Phys. Rev. B 93, 085105 (2016)

[26] R. Fleury, A. Khanikaev, and A. Alu, arXiv: 1511.08427.

[27] S. Malzard, C. Poli, and H. Schomerus, Phys. Rev. Lett. 115, $200402(2015)$

[28] H. Schomerus, Opt. Lett. 38, 1912 (2013).

[29] C. Poli, M. Bellec, U. Kuhl, F. Mortessagne, and H. Schomerus, Nat. comm. 6 (2015).

[30] J. M. Zeuner, M. C. Rechtsman, Y. Plotnik, Y. Lumer, S. Nolte, M. S. Rudner, M. Segev, and A. Szameit, Phys. Rev. Lett. 115, 040402 (2015)

[31] A. Russomanno and E. G. Dalla Torre, arXiv: 1510.08866 .

[32] R. Bücker, J. Grond, S. Manz, T. Berrada, T. Betz, C. Koller, U. Hohenester, T. Schumm, A. Perrin, and J. Schmiedmayer, Nat. Phys. 7, 608 (2011).

[33] C. Emary and T. Brandes, Phys. Rev. Lett. 90, 044101 (2003).

[34] A. J. Heeger, S. Kivelson, J. R. Schrieffer, and W. P. Su, Rev. Mod. Phys. 60, 781 (1988).

[35] The derivation of the effective Hamiltonian and its validity can be found in the supplemental materials.

[36] A. Altland and M. R. Zirnbauer, Phys. Rev. B 55, 1142 (1997)

[37] V. Gurarie, Phys. Rev. B 83, 085426 (2011).

[38] J. Colpa, Physica A 93, 327 (1978).

[39] Arnold, Mathematical Methods of Classical Mechanics (Springer-Verlag, New York, 1978).

[40] A. R. Kolovsky, H. J. Korsch, and E.-M. Graefe, Phys. Rev. A 80, 023617 (2009).

[41] V. Starzhinskii and V. Yakubovich, Linear differential equations with periodic coefficients (Wiley London, 1975).

[42] J. Tomkovič, W. Muessel, H. Strobel, S. Löck, P. Schlagheck, R. Ketzmerick, and M. K. Oberthaler, arXiv: 1509.01809 .

\section{Supplementary Information}

\section{Derivation of the effective Hamiltonian}

In order to efficiently determine the full parameter range where unstable midgap states can be expected, we derive a time-independent effective Hamiltonian. We generalize the procedure of Ref. [23] to Bogoliubov Hamiltonians which, to our knowledge, has not been done before.

The equation of motion can be written as

$$
i \frac{d}{d t}\left|\Psi_{k}\right\rangle=\boldsymbol{\sigma}_{\boldsymbol{z}}\left(\mathbf{H}_{k, 0}+\mathbf{H}_{k, 1} \cos \Omega t\right)\left|\Psi_{k}\right\rangle,
$$

where

$$
\begin{aligned}
& \mathbf{H}_{k, 0}=\mathbb{1} \otimes\left[-\mu \mathbb{1}+h_{x, 0}(k) \sigma_{x}+h_{y, 0}(k) \sigma_{y}\right]+g \sigma_{x} \otimes \mathbb{1} \\
& \mathbf{H}_{k, 1}=\mathbb{1} \otimes\left[h_{x, 1}(k) \sigma_{x}+h_{y, 1}(k) \sigma_{y}\right] .
\end{aligned}
$$

In the following, we suppress the dependence of momentum $k$ in the coefficients $h_{x, 0}, h_{y, 0}, h_{x, 1}, h_{y, 1}$ for a notational reason. We transform this equation into an interaction picture. This is defined by the unitary transformation

$$
\mathbf{U}_{k, \alpha, \beta}(t)=\exp \left[-i \boldsymbol{\sigma}_{\boldsymbol{z}} \frac{\mathbf{H}_{k, 1}}{\sqrt[4]{\operatorname{det} \mathbf{H}_{k, 1}}} \theta_{\alpha}(t),\right] \exp \left[-i \boldsymbol{\sigma}_{\boldsymbol{z}} \frac{\beta \Omega t}{2}\right],
$$


where

$$
\theta_{k, \alpha}(t)=\sqrt[4]{\operatorname{det} \mathbf{H}_{k, 1}} \frac{\sin \Omega t}{\Omega}+\frac{\alpha \Omega}{2} t
$$

The parameters $\alpha, \beta$ are integers. In the interaction picture, the equation of motion reads

$$
i \frac{d}{d t}\left|\Psi_{k, I}\right\rangle=\mathbf{U}_{k, \alpha, \beta}^{\dagger}(t) \boldsymbol{\sigma}_{\boldsymbol{z}}\left(\mathbf{H}_{k, 0}-\alpha \frac{\Omega}{2} \frac{\mathbf{H}_{k, 1}}{\sqrt[4]{\operatorname{det} \mathbf{H}_{k, 1}}}-\frac{\beta \Omega}{2}\right) \mathbf{U}_{k, \alpha, \beta}(t)\left|\Psi_{k, I}\right\rangle .
$$

The integer $\alpha$ can be chosen to effectively reduce the gap between the two bands and the integer $\beta$ shifts the chemical potential $\mu$ which can be understood later by considering Eqs. 18) and (27). First, we calculate an explicit expression for $\mathbf{U}_{k, \alpha, \beta}(t)$. Defining $\phi_{k} \equiv \operatorname{Im} \ln \left(h_{x, 1}+i h_{y, 1}\right)$, we obtain

$$
\mathbf{U}_{k, \alpha, \beta}(t)=\left(\begin{array}{cc}
U_{k, \alpha, \beta} e^{-i \frac{\beta \Omega}{2} t} & 0 \\
0 & U_{k, \alpha, \beta}^{\dagger} e^{i \frac{\beta \Omega}{2} t}
\end{array}\right)
$$

where

$$
U_{k, \alpha, \beta}(t)=\mathbb{1} \cos \theta_{k, \alpha}(t)+i \sigma_{x} \sin \theta_{k, \alpha}(t) \cos \phi_{k}+i \sigma_{y} \sin \theta_{k, \alpha}(t) \sin \phi_{k}
$$

For a notational reason we define

$$
h_{\eta}^{\alpha}=h_{\eta, 0}-\frac{\alpha \Omega}{2} \frac{h_{\eta, 1}}{\sqrt[4]{\operatorname{det} \mathbf{H}_{k, 1}}} \quad \text { with } \quad \eta=x, y
$$

where we again suppress the argument $k$. We evaluate the matrix product

$$
\begin{aligned}
& U_{k, \alpha, \beta}^{\dagger}\left[h_{x}^{\alpha} \sigma_{x}+h_{y}^{\alpha} \sigma_{y}\right] U_{k, \alpha, \beta}= \\
& =\sigma_{x}\left[\cos ^{2} \theta_{k, \alpha}(t) h_{x}^{\alpha}+\sin ^{2} \theta_{k, \alpha}(t)\left(h_{x}^{\alpha} \cos ^{2} \phi_{k}-h_{x}^{\alpha} \sin ^{2} \phi_{k}+2 h_{y}^{\alpha} \cos \phi_{k} \sin \phi_{k}\right)\right] \\
& +\sigma_{y}\left[\cos ^{2} \theta_{k, \alpha}(t) h_{y}^{\alpha}+\sin ^{2} \theta_{k, \alpha}(t)\left(h_{y}^{\alpha} \sin ^{2} \phi_{k}-h_{y}^{\alpha} \cos ^{2} \phi_{k}+2 h_{x}^{\alpha} \cos \phi_{k} \sin \phi_{k}\right)\right] \\
& +\left(h_{y}^{\alpha} \cos \phi_{k}-h_{x}^{\alpha} \sin \phi_{k}\right) 2 \sin \theta_{k, \alpha}(t) \cos \theta_{k, \alpha}(t) \sigma_{z} \\
& \equiv \tilde{h}_{x}^{\alpha}(t) \sigma_{x}+\tilde{h}_{y}^{\alpha}(t) \sigma_{y}+\tilde{h}_{z}^{\alpha}(t) \sigma_{z} .
\end{aligned}
$$

Finally we apply a rotating-wave approximation by a time average of the coefficients. In doing so we use that

$$
\begin{aligned}
& \frac{1}{2 \pi / \Omega} \int_{0}^{2 \pi / \Omega} \cos ^{2} \theta_{k, \alpha}(t)=\frac{1}{2}\left[1+\mathcal{J}_{\alpha}\left(\frac{2 \sqrt[4]{\operatorname{det} \mathbf{H}_{k, 1}}}{\Omega}\right)\right], \\
& \frac{1}{2 \pi / \Omega} \int_{0}^{2 \pi / \Omega} \sin ^{2} \theta_{k, \alpha}(t)=\frac{1}{2}\left[1-\mathcal{J}_{\alpha}\left(\frac{2 \sqrt[4]{\operatorname{det} \mathbf{H}_{k, 1}}}{\Omega}\right)\right], \\
& \frac{1}{2 \pi / \Omega} \int_{0}^{2 \pi / \Omega} \sin \theta_{k, \alpha}(t) \cos \theta_{k, \alpha}(t)=0,
\end{aligned}
$$

where $\mathcal{J}_{\alpha}(x)$ denotes the Bessel function of order $\alpha$. Analogously, we treat the term

$$
U_{k, \alpha, \beta}^{\dagger}(t) g \mathbb{1} U_{k, \alpha, \beta}^{\dagger}(t)
$$

Finally, we obtain

$$
\begin{aligned}
\mathbf{H}_{\mathrm{eff}, k} & =\mathbb{1} \otimes \vec{h}_{\mathrm{eff}}^{\alpha}(k) \vec{\sigma}-\mathbb{1} \otimes \mu_{\mathrm{eff}}^{\beta} \mathbb{1} \\
& +\sigma_{x} \otimes \vec{G}_{\mathrm{eff}}^{\alpha, \beta}(k) \vec{\sigma}+\sigma_{x} \otimes g_{\mathrm{eff}}^{\alpha, \beta}(k) \mathbb{1}
\end{aligned}
$$


with the coefficients

$$
\begin{aligned}
h_{\mathrm{eff}, x}^{\alpha}(k) & =h_{x}^{\alpha}\left[f_{k}^{+}\left(\frac{h_{y}^{\alpha}}{h_{x}^{\alpha}}\right)+f_{k}^{-}\left(\frac{h_{y}^{\alpha}}{h_{x}^{\alpha}}\right) \mathcal{J}_{\alpha}\left(\frac{2 \sqrt[4]{\operatorname{det} \mathbf{H}_{k, 1}}}{\Omega}\right)\right] \\
h_{\mathrm{eff}, y}^{\alpha}(k) & =h_{y}^{\alpha}\left[f_{k}^{-}\left(-\frac{h_{x}^{\alpha}}{h_{y}^{\alpha}}\right)+f_{k}^{+}\left(-\frac{h_{x}^{\alpha}}{h_{y}^{\alpha}}\right) \mathcal{J}_{\alpha}\left(\frac{2 \sqrt[4]{\operatorname{det} \mathbf{H}_{k, 1}}}{\Omega}\right)\right], \\
\mu_{\mathrm{eff}}^{\beta} & =\mu-\beta \frac{\Omega}{2}, \\
g_{\mathrm{eff}}^{\alpha, \beta}(k) & =\frac{g}{2}\left[J_{-\beta-\alpha}\left(\frac{\left.\left.2 \sqrt[4]{\operatorname{det} \mathbf{H}_{k, 1}}\right)+J_{\beta-\alpha}\left(\frac{2 \sqrt[4]{\operatorname{det} \mathbf{H}_{k, 1}}}{\Omega}\right)\right]}{\Omega}\right)-J_{\beta-\alpha}\left(\frac{2 \sqrt[4]{\operatorname{det} \mathbf{H}_{k, 1}}}{\Omega}\right)\right] \\
G_{\mathrm{eff}, x}^{\alpha, \beta}(k) & =\frac{g}{2} \cos \phi\left[J_{-\beta-\alpha}\left(\frac{\sqrt[4]{\operatorname{det} \mathbf{H}_{k, 1}}}{\Omega}\right)\right] \\
G_{\mathrm{eff}, y}^{\alpha, \beta}(k) & =\frac{g}{2} \sin \phi\left[J_{-\beta-\alpha}\left(\frac{2 \sqrt[4]{\operatorname{det} \mathbf{H}_{k, 1}}}{\Omega}\right)-J_{\beta-\alpha}\left(\frac{2 \sqrt[4]{\operatorname{det} \mathbf{H}_{k, 1}}}{\Omega}\right)\right]
\end{aligned}
$$

where we have defined

$$
2 f_{k}^{ \pm}(x) \equiv 1 \pm \cos ^{2} \phi_{k} \mp \sin ^{2} \phi_{k} \pm 2 x \sin \phi_{k} \cos \phi_{k}
$$

This expression is valid if $h_{x}^{\alpha}, h_{y}^{\alpha}, \mu_{\mathrm{eff}}^{\beta}, g \ll \Omega$. In order to fulfill $\mu_{\text {eff }}^{\beta} \ll \Omega$, we choose $\beta$ so that $\left|\mu_{\text {eff }}^{\beta}\right|$ is minimal, thus $\left|\mu_{\mathrm{eff}}^{\beta}\right|<\Omega / 4$. In a similar way we have to choose $\alpha$, so that the modulus of $h_{x}^{\alpha}, h_{y}^{\alpha}$ defined in Eq. 18 are as small as possible. The effective Hamiltonian still fulfills a generalized chiral symmetry relation (4) in the letter, independently for all momenta $k$. The Hamiltonian Eq. (24) can be diagonalized so that we obtain the energies

$$
\begin{aligned}
& \epsilon_{ \pm}= \pm \sqrt{\left|\vec{h}_{\mathrm{eff}}^{\alpha}\right|^{2}+\left(\mu_{\mathrm{eff}}^{\beta}\right)^{2}-\left(g_{\mathrm{eff}}^{\beta}\right)^{2}-\left(\tilde{g}_{1}^{\beta}\right)^{2} \pm 2 \sqrt{A}} \\
& A=-\tilde{g}_{1}^{\beta}\left|\vec{h}_{\mathrm{eff}}^{\alpha}\right|\left(\tilde{g}_{1}^{\beta}\left|\vec{h}_{\mathrm{eff}}^{\alpha}\right| \sin ^{2} \delta \phi+\tilde{g}_{\mathrm{eff}}^{\beta} \mu^{\beta} \sin ^{2} \frac{\delta \phi}{2}\right)+\left(g_{\mathrm{eff}}^{\beta} \tilde{g}_{1}^{\beta}+\left|\vec{h}_{\mathrm{eff}}^{\alpha}\right| \mu^{\beta}\right)^{2} .
\end{aligned}
$$

where $\delta \phi$ is the angle between $\vec{G}_{\text {eff }}^{\beta}$ and $\vec{h}_{\text {eff }}$. The $\epsilon_{ \pm}$resemble the actual quasienergies of the system modulo $\Omega / 2$ as a consequence of Eq. 27 shifting the chemical potential, and the folding of the quasienergies into the window $(-\Omega / 2, \Omega / 2)$. We compare the analytical calculated $\epsilon_{ \pm}$and the numerical quasienergies in Fig. 1(b) and (c), where we choose $\alpha=0$ and $\beta=-2$ so that the condition of validity is fulfilled. The effective Hamiltonian reproduces all features of our numerics, especially the instabilities, although we work with a mediate frequency $\Omega$. Corresponding to the actual quasienergies, the system is stable if all $\epsilon_{ \pm}$are real valued. In Fig. 2(a) we use the $\epsilon_{ \pm}$to calculate the regions of global stability. The result is depicted with the black dashed lines. 